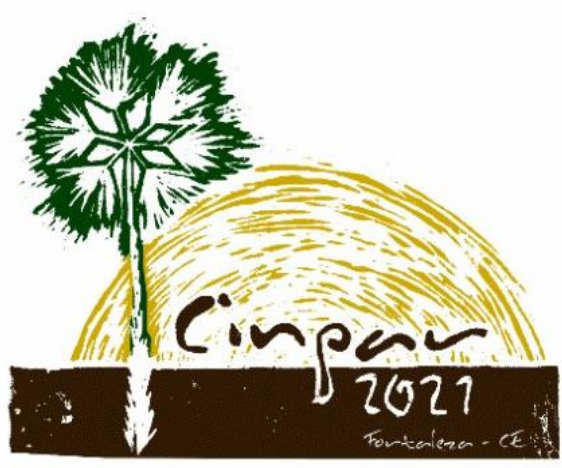

XVII Congresso Internacional sobre Patologia e

Reabilitação das Construções

XVII Congreso Internacional sobre Patología y Rehabilitación de las Construcciones

XVII International Conference on Pathology and Constructions Rehabilitation

FORTALEZA (Brasil), 3 a 5 de junho de 2021

https://doi.org/10.4322/CINPAR.2021.014

\title{
Diferenças nos padrões de identificação termográfica de vários materiais e defeitos
}

\section{Differences in patterns of thermographic identification of various materials and defects}

\author{
Raynnara LUCENAS ${ }^{1}$, Elton BAUER ${ }^{2}$, Elier PAVÓN ${ }^{3}$ \\ ${ }^{1}$ Universidade de Brasília, Brasília, Brasil, lucenasraynnara@gmail.com1 \\ 2 Universidade de Brasília, Brasília, Brasil, elbauerlem@gmail.com 2 \\ ${ }^{3}$ Universidade Federal do Oeste da Bahia, Barreiras, Brasil, elierpavon@gmail.com 3
}

\begin{abstract}
Resumo: Uma forma de avaliar materiais e componentes com o intuito de identificar e caracterizar as anomalias e defeitos ocultos é a termografia de infravermelho. Esta técnica que consiste em um método não destrutivo é aplicada com base em termogramas obtidos a partir de medições em tempo real e sem contato. A radiação infravermelha é medida pela câmera termográfica e com base em informações de emissividade, temperatura aparente refletida e outras grandezas se consegue obter o mapeamento de temperaturas em uma imagem. $O$ estudo dos termogramas mostra a distribuição de temperaturas na superfície do objeto alvo, o que permite associar diferenças mensuradas a padrões de falha, e também característicos de diferentes materiais do elemento construtivo. O presente estudo tem por objetivo investigar a aplicação da termografia na detecção de defeitos e particularizar comportamentos de diferentes materiais inseridos em um sistema de revestimento. Para isto se emprega a termografia ativa, onde a placa com seis defeitos inseridos dos materiais poliestireno expandido, cerâmica, concreto, madeira e vazio, com revestimento em argamassa será exposta à fontes de aquecimento em ciclo direto. A investigação das estapas de aquecimento e arrefecimento é realizada em laboratório sob condições controladas, para identificação dos comportamentos das temperaturas nos defeitos empregando a termografia qualitativa e quantitativa. Se avalia a aptidão da função de contraste termográfico absoluto na identificação dos defeitos ao longo do processo. Como resultado se tem os valores de Delta-T dos diferentes defeitos inseridos internamente na placa de estudo, o que permite aumentar a precisão de análise para aplicações em campo. Também se observa a evolução desse comportamento como decorrer do aquecimento e arrefecimento, identificando situações de maior precisão para identificação dos defeitos por meio da aplicação da termografia infravermelha.
\end{abstract}

Palavras-chave: Termografia. Argamassa de Revestimento. Defeitos. Falhas.

\begin{abstract}
One way of evaluating materials and components in order to identify and characterize hidden anomalies and defects is infrared thermography. This technique, which consists of a non-destructive method, is applied based on thermograms obtained from measurements in real time and without contact. Infrared radiation is measured by the thermographic camera and based on information on emissivity, reflected apparent temperature and other quantities, it is possible to obtain the mapping of temperatures in an image. The study of thermograms shows the distribution of temperatures on the surface of the target object, which allows associating measured differences with failure patterns, as well as characteristics of different materials of the construction element. The present study aims to investigate the application of thermography in the
\end{abstract}


detection of defects and to detail the behavior of different materials inserted in a coating system. For this purpose, active thermography is used, where the plate with six defects inserted from the expanded polystyrene, ceramic, concrete, wood and empty materials, with mortar coating will be exposed to heating sources in a direct cycle. The investigation of the heating and cooling steps is carried out in the laboratory under controlled conditions, to identify the behavior of the temperatures in the defects using qualitative and quantitative thermography. The suitability of the absolute thermographic contrast function is assessed in the identification of defects throughout the process. As a result, the Delta-T values of the different defects are inserted inside the study board, which allows to increase the analysis accuracy for field applications. It is also possible to observe the evolution of this behavior as a result of heating and cooling, identifying situations of greater precision for the identification of defects through the application of infrared thermography.

Keywords: Thermography. Rendering Mortar. Defects. Faults.

\section{Introdução}

As edificações apresentam ao longo dos anos de vida útil um processo natural de envelhecimento resultante da interação entre o edifício e os agentes de degradação, que podem ser de natureza mecânica, eletromagnéticos, térmicos, químicos ou biológicos (ZANONI, 2015; ISO 15868-2, 2012). No estudo do comportamento das fachadas de edifícios, a análise das respostas frente a ação de agentes de degradação, assim como a presença de defeitos principalmente na forma de elementos ocultos, tem se tornado possível através do desenvolvimento de técnicas que possibilitam tais análises.

A termografia de infravermelho consiste em uma técnica que permite a investigação detalhada das temperaturas de um termograma a partir dos padrões de comportamento identificados, possibilitando a detecção e caracterização de defeitos. Os termogramas são obtidos a partir de medições em tempo real e sem contato, com base na transformação da radiação infravermelho emitida pelo objeto alvo na faixa infravermelha do espectro eletromagnético, em níveis visíveis de imagem (MADRUGA et al., 2010; MALDAGUE, 2001).

A termografia pode ser aplicada utilizando a técnica passiva quando nenhuma estimulação artificial é empregada sobre o objeto alvo para gerar diferenças de temperatura entre este e o meio envolvente e pode ser ativa quando a fonte de calor é artificial para gerar o contraste. Dentre os diferentes tipos tem se o pulso, ciclos de pulsos, vibro-termografia, clicos de calor, dentre outros, constituindo um aquecimento por pulsação ou gradual (PEDRA, 2011; BAUER e PAVÓN, 2015). Dentre as formas de análises dos termogramas tem se a qualitativa que consiste em observar as diferenças de temperatura para identificar a região crítica, ou seja, as regiões que aparecem mais quentes ou frias quando comparadas a uma situação padrão, e quantitativa quando se faz uma análise das temperaturas obtidas com o objetivo de determinar a severidade das anomalias e defeitos. As funções de contraste são utilizadas como ferramentas de análise dos termogramas, conferindo precisão aos resultados e eliminando os ruídos (SILVA, 2012; PEDRA, 2011).

A análise dos termogramas permite observar os padrões de falhas e sua severidade. Dentre as anomalias avaliadas por meio da termografia infravermelha quando empregadas na inspeção de fachadas se pode mencionar descolamentos, fissuras, umidades, presença de vazios e dentre outros. As investigações de laboratório tendem a focar suas análises em defeitos presentes em materiais e elementos.

As fachadas das edificações constituem um sistema de vedação vertical, que possuem no seu interior diferentes elementos como estrutura do edifício em concreto armado e na superfície materiais e elementos como revestimentos e esquadrias. Estes materiais com diferentes propriedades térmicas podem gerar pertubações nos termogramas e por isso a necessidade de estudar seus padrões de temperartura (PAVÓN, 2017).

A identificação de defeitos consiste na observação dos comportamentos de temperaturas, diferentes de determinada situação padrão, para um determinado material analisado. Estes defeitos são constituídos de vazios ou materiais diferentes que apresentam propriedades térmicas distintas e com isso nos termogramas resultam em comportamentos de temperatura divergentes. A depender das propriedades térmicas, estes defeitos podem facilitar ou dificultar a trasferência de calor de uma superfície a outra do material ou elemento em que estão inseridos, perturbando o comportamento de termperaturas esperado. $\mathrm{Na}$ avaliação 
dos defeitos inseridos em placa, este estudo realizado em laboratório tem como intuito a análise da distribuição de temperaturas nos termogramas, sendo considerado o ciclo de aquecimento e arrefecimento, permitindo um estudo mais sensível quanto ao comportamento e identificação dos diferentes materiais (BAUER, 2015).

Diferentes estudos são realizados na área de engenharia civil para deteç̧ão de defeitos utilizando a termografia infravermelha. Na detecção de descolamentos cerâmicos em fachadas de edifícios destaca-se as investigações realizadas por Edis et. al. (2015), Bauer et. al. (2016) e Pavón (2017); na identificação de presença de umidade em edifícios tem-se Edis, et. al. (2014) e Barreira, et. al. (2016) e na deteç̧ão fissuras tem-se as investigações de Bauer et. al. (2016) e Milhomem (2019).

Dessa forma, neste estudo o objetivo é investigar a aplicação da termografia ativa de pulso longo, assim como a análise qualitativa e quantitativa na detecção de defeitos devido à presença de elementos ocultos (poliestireno expandido, cerâmica, concreto, madeira e vazio), inseridos em placa com revestimento em argamassa e particularizar os comportamentos dos diferentes materiais com base nos termogramas obtidos e na aplicação da função de constraste absoluto.

\section{Metodologia}

\subsection{Corpo de prova e defeitos}

A metodologia experimental empregada para investigar o comportamento das temperaturas dos elementos ocultos inseridos na placa consiste no emprego da termografia infravermelha de pulso longo, estudando o aquecimento e arrefecimento. Para isto foi moldada em laboratório uma placa em argamassa com dimensões $50 \times 25 \times 4 \mathrm{~cm}$, sendo inseridos seis tipos de materiais como elementos ocultos (defeitos), sendo eles o poliestireno expandido, cerâmica, concreto, madeira e um vazio. O quadro 1 apresenta as placas P1 com as posições e tipos de defeitos e a placa P1 com uma camada de revestimento em argamassa de cimento e areia artificial, com espessura de $2,5 \mathrm{~mm}$. Vale ressaltar que esta argamassa foi utilizada para moldar e preencher a placa. A placa P1 com revestimento foi a utilizada neste estudo para obtenção dos termogramas.

Os diferentes materiais com suas respectivas propriedades térmicas tendem a alterar o comportamento de distribuição de temperarturas e evidenciar uma possível anomalia térmica no elemento. $\mathrm{O}$ quadro 2 apresenta a caracterização dos materiais quanto as suas propriedades térmicas de condutividade térmica e calor específico. Esta caracterização é utilizada como parâmetro na interpretação do comportamento das temperaturas dos termogramas, sendo esperado que a condutividade térmica influencie na capacidade de identificação dos materiais inseridos como elementos ocultos. O defeito denominado como vazio não é considerado no quadro uma vez que na placa resulta em uma maior profundidade na placa de argamassa.

Quadro 1 - Características dos elementos ocultos (defeitos) inseridos nas placas (Adaptado de PAVÓN, 2017)

\begin{tabular}{|c|c|c|c|c|}
\hline $\begin{array}{c}\text { Corpo de } \\
\text { prova (Placa) }\end{array}$ & Imagem digital & Vista em plant & Vista lateral & $\begin{array}{c}\text { Materiais dos } \\
\text { defeitos }\end{array}$ \\
\hline P1 & E & 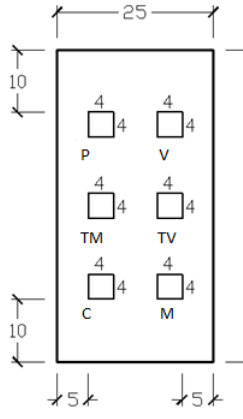 & 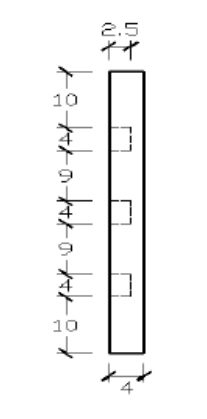 & $\begin{array}{l}\text { P-Poliestireno } \\
\text { expandido } \\
\text { V- Vazio } \\
\text { TM- Tijolo } \\
\text { Maciço } \\
\text { TV- Bloco } \\
\text { cerâmico } \\
\text { C- Concreto } \\
\text { M- Madeira }\end{array}$ \\
\hline
\end{tabular}




\begin{tabular}{|c|c|c|c|c|c|}
\hline P1 com \\
revestimento
\end{tabular}$\quad+45$ -

Quadro 2 - Caracterização dos materiais dos defeitos inseridos e da argamassa utilizada (ABNT NBR 15220:2005)

\begin{tabular}{|c|c|c|}
\hline Materiais & $\begin{array}{c}\text { Condutividade } \\
\text { Térmica } \Lambda(W /(m . K))\end{array}$ & $\begin{array}{c}\text { Calor específico } \\
\mathrm{c}(\mathrm{kJ} /(\mathrm{kg} . \mathrm{K}))\end{array}$ \\
\hline Argamassa (referência) & 1.15 & 1.00 \\
\hline Concreto & 1.75 & 1.00 \\
\hline Cerâmica & 0.90 & 0.92 \\
\hline Madeira (pinus) & 0.15 & 1.34 \\
\hline Poliestireno expandido & 0.04 & 1.42 \\
\hline
\end{tabular}

2.2 Metodologia experimental de obtenção dos termogramas

A aplicação da termografia infravermelha de pulso longo é realizada considerando dois ciclos, o primeiro ciclo de aquecimento consiste no aquecimento gradual da placa por meio de seis lâmpadas infravermelhas de $250 \mathrm{~W}$ cada e o segundo ciclo de arrefecimento onde ocorre o desligamento da fonte artificial de aquecimento e resulta no resfriamento gradual da placa. A disposição dos aparatos do experimento pode ser observada na figura 1 (a), onde a câmera infravermelha distancia-se da placa em 1.70 metros e o sistema de aquecimento constituido de três lâmpadas infravermelhas cada, está situado a 0.90 metros da placa de argamassa que pode ser observado na imagem digital da figura 1 (b). Vale ressaltar que este aparato de ensaio experimental desenvolvido por Pavón (2017) que investigou em laboratório a presença de defeitos em placas e em protótipos de paredes, tem sido aplicado em vários estudos de termografia em laboratório como a investigação do comportamento de fissuras em placas desenvolvido por Milhomem (2019), a análise da influência do ângulo de obtenção dos termogramas nas temperaturas obtidas observado por Aidar et. al. (2018) e dentre outros.
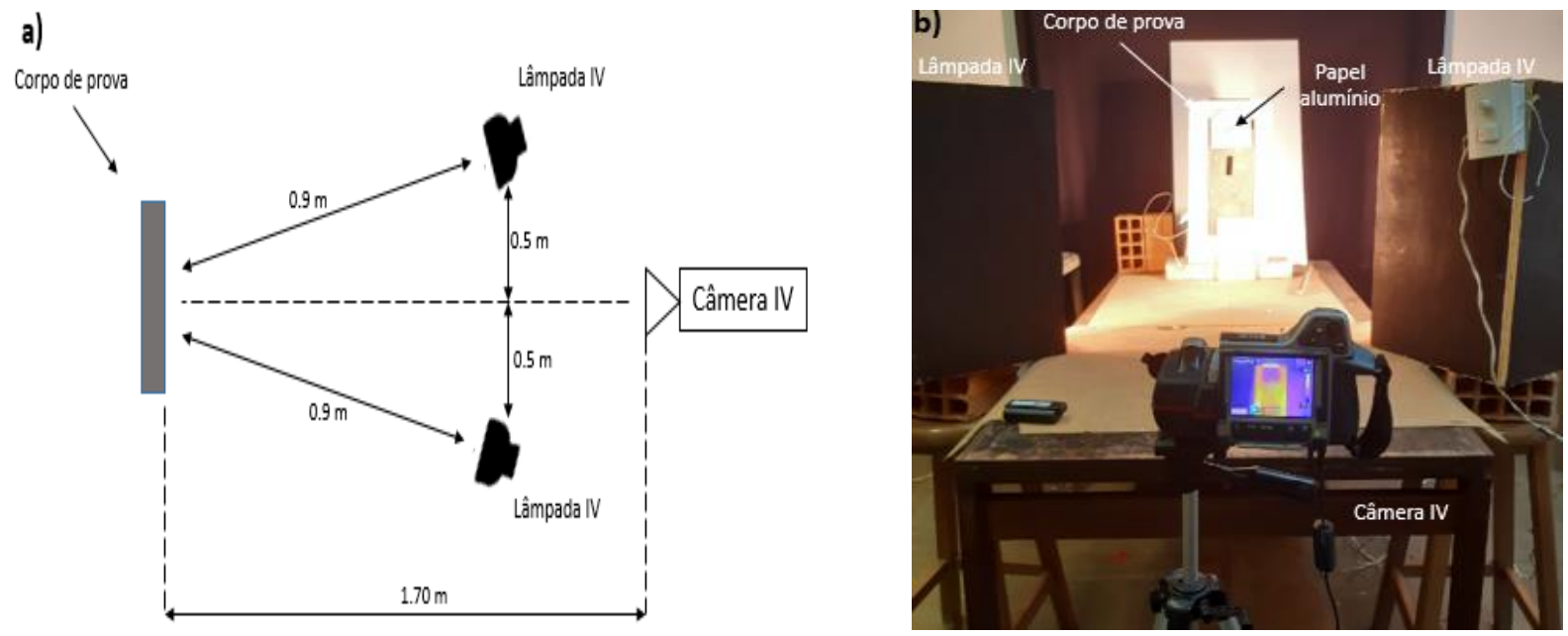

Figura 1 - Metodologia utilizada (a) obtenção dos termogramas (b) imagem digital do aquecimento da placa com elementos ocultos (Autores). 
Na figura 1 (b) é possível observar no corpo de prova uma região contendo papel alumínio corrugado e logo abaixo uma faixa de fita preta, sendo estes utilizados para determinação da temperatura aparente refletida segundo a ASTM E1933-14 e emissividade segundo a ASTM E1862-14, respectivamente.

Para obtenção dos termogramas utiliza-se uma câmera infravermelha modelo FLIR T400, com faixa de temperatura variando de $20^{\circ} \mathrm{C}$ a $120^{\circ} \mathrm{C}$, com precisão de $2 \%$, resolução de $320 \times 240$ pixels, com faixa espectral de 7,5 a $13 \mu \mathrm{m}$, lentes de 25 graus e IFOV de 1,363 mrad. Para a definição dos parâmetros de temperatura ambiente e umidade relativa se utiliza um psicrômetro de umidade sem fuso com termômetro modelo MO297.

O ciclo de aquecimento tem duração de 120 minutos com registros de imagens termográficas a cada 4 minutos, assim como o ciclo de arrefecimento tem duração de 120 minutos com registros a cada 4 minutos, resultando no período total de $\mathbf{2 4 0}$ minutos de experimentos e obtenção de 60 termogramas. Os dados de emissividade, temperatura ambiente e umidade relativa são inseridos na câmera infravermelha e posteriormente a obtenção das imagens térmicas, se utiliza o software QuickReport 1.2 para análise quantitativa dos termogramas.

A análise qualitativa consiste na observação dos comportamentos de temperatura com base nas diferenças de tonalidades (regiões mais quentes ou mais frias) nos termogramas. Já a análise quantitativa consiste na mensuração da temperatura ao longo da placa com o objetivo de identificar a presença de comportamentos anormais de temperatura. Neste estudo através do software QuickReport 1.2 se utiliza as ferramentas área para observar o comportamento de temperatura sobre a superfície onde os defeitos estão posicionados e a ferramenta linha para determinação da temperatura da região sem defeito. O conhecimento das temperaturas destas regiões permite a determinação do valor do contraste absoluto ou Delta-T para cada elemento oculto, segundo a equação 1 (MALDAGUE, 2001; BAUER e PAVÓN, 2015; PAVÓN, 2017) . A finalidade de utilização do Delta-T é identificar os momentos de melhor visualização dos defeitos (BAUER, et al., 2016) e a influência das propriedades térmicas dos materiais inseridos na placa no comportamento de temperatura dos defeitos.

$$
\text { Delta }-T=T_{a}-T_{r}
$$

Sendo Delta-T o contraste obtido a partir da diferença entre a temperatura do defeito $\left(T_{a}\right)$ e a temperatura da região vizinha ao defeito $\left(T_{r}\right)$.

\section{Resultados}

Os termogramas obtidos nesta investigação consideram um ciclo direto de aquecimento e arrefecimento, ou seja a fonte de calor artificial encontra-se posicionada na superfície frontal do corpo de prova (placa). 0 experimento tem a duração total de 240 minutos, com registros de imagens térmicas a cada 4 minutos. 0 ciclo de aquecimento com duração de 120 minutos, apresenta conforme a figura 2 (a) os resultados de DeltaT para cada elemento oculto inserido na placa e a figura 2 (b) apresenta os resultados de Delta-T para o ciclo de arrefecimento, também com duração de 120 minutos.

Os valores de Delta-T são resultados da aplicação do contraste absoluto entre a região com presença de elementos ocultos e a região sem defeitos, sendo seus valores analisados como módulo . É possível observar o comportamento das temperaturas dos diferentes materiais no aquecimento e arrefecimento. Observa-se que no aquecimento a madeira (M) apresenta os maiores resultados de Delta-T, seguida do vazio (V), poliestireno expandido ( $P$ ), tijolo cerâmico vazado (TV), tijolo cerâmico maciço (TM) e concreto (C), respectivamente. Com a mudança do ciclo de aquecimento para arrefecimento, ocorrem alterações nos resultados de Delta-T, isto acontece pela interrupção do fornecimento de calor e alteração no fluxo de calor da placa. Neste momento os defeitos ao invés de se apresentarem mais quentes que a argamassa da placa, se mostram mais frios. Considerando os resultados do Delta-T em módulo, observa-se que o poliestireno expandido $(P)$ apresenta os maiores contrastes nos resultados de Delta-T, seguido do vazio (V), madeira (M), tijolo cerâmico maciço (TM), tijolo cerâmico vazado (TV) e concreto (C). 

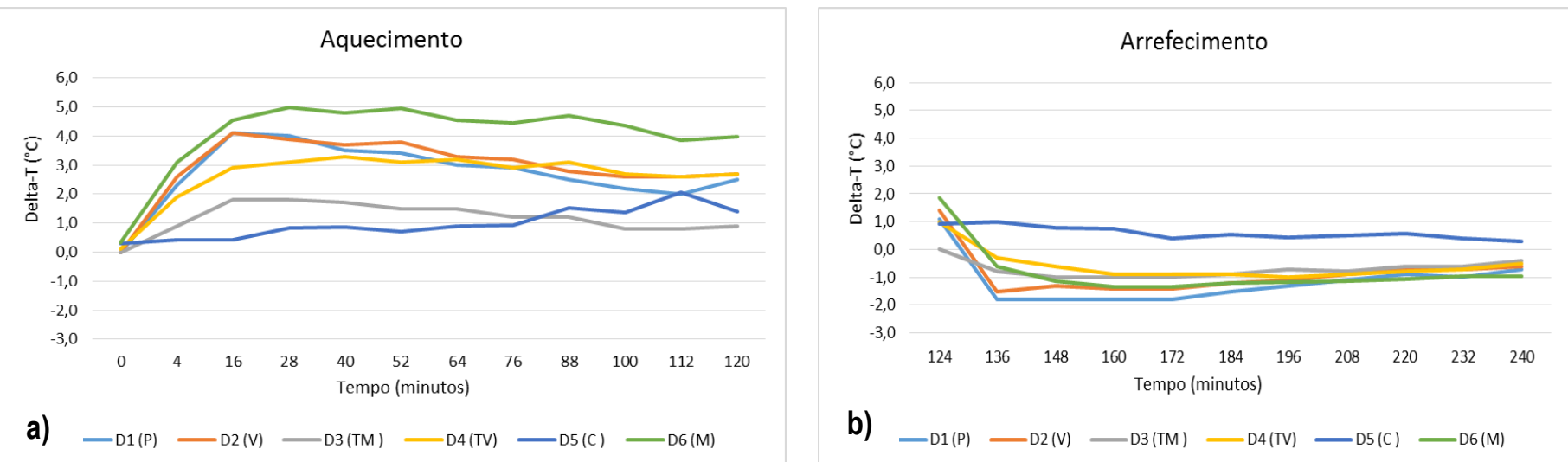

Figura 2 - Gráfico do Delta-T dos elementos ocultos D1 (Poliestireno), D2 (Vazio), D3 (Tijolo maciço - cerâmico), D4 (Tijolo vazado - cerâmico), D5 (Concreto) e D6 (Madeira) no ciclo (a) aquecimento e (b) arrefecimento (Autores).

O elemento oculto em concreto $(\mathrm{C})$ apresenta uma certa estabilidade de variações de Delta-T, considerando os ciclos de aquecimento e arrefecimento, apresentando um pico próximo a $2^{\circ} \mathrm{C}$ ao final do ciclo de aquecimento e ao longo de todo o experimento, sua variação foi de 0 a $2^{\circ} \mathrm{C}$. Esta menor variação explica-se pelas propriedades térmicas semelhantes a da argamassa de revestimento. Observa-se também que ao longo do aquecimento os valores de Delta-T para todos os elementos ocultos, exceto o concreto (C), apresentam queda, o que pode ser resultado da evolução do fluxo de calor. É possível observar que a transição do ciclo de aquecimento para o arrefecimento, corresponde a uma alteração nos valores de Delta-T, pela alteração na dinâmica do fluxo de calor entre a argamassa e os elementos ocultos inseridos.

Estes resultados permitem apontar a tendência de que maiores contrastes de temperatura são obtidos para os materiais com menor condutividade térmica, sendo no aquecimento os máximos valores de Delta-T obtidos para a madeira $\left(5,0^{\circ} \mathrm{C}\right)$, seguida do poliestireno $\left(4,1^{\circ} \mathrm{C}\right)$, vazio $\left(4,1^{\circ} \mathrm{C}\right)$, tijolo cerâmico vazado $\left(3,3^{\circ} \mathrm{C}\right)$, concreto $\left(2,1^{\circ} \mathrm{C}\right)$ e tijolo cerâmico maciço $\left(1,8^{\circ} \mathrm{C}\right)$ e no arrefecimento os máximos valores de Delta-T são para a madeira $\left(1,9^{\circ} \mathrm{C}\right)$, poliestireno $\left(1,8^{\circ} \mathrm{C}\right)$, vazio $\left(1,5^{\circ} \mathrm{C}\right)$, tijolo cerâmico vazado $\left(1,0^{\circ} \mathrm{C}\right)$, concreto $\left(1,0^{\circ} \mathrm{C}\right)$ e tijolo cerâmico maciço $\left(1,0^{\circ} \mathrm{C}\right)$, sendo os menores valores de Delta-T obtidos para os materiais com maior condutividade térmica. Isto ocorre pois com menor coeficiente de condutividade térmica, maior será a dificuldade do fluxo transportar calor da superfície aquecida a outra superfície da placa na região do defeito, quando no aquecimento e com isso o fluxo é desacelerado e permanece concentrado na região de superfície acima do defeito. No arrefecimento ocorre a inversão do fluxo e com isso o elemento oculto apresenta-se como uma resistência à saída de calor, com isso a superfície localizada a cima da posição do defeito tende a apresentar-se mais fria que a região em argamassa de preenchimento. Quanto maior for a condutividade térmica do material, este se apresentará mais quente no arrefecimento quando comparado a materiais com menor condutividade que tendem a apresentar-se mais frios. Este comportamento é evidenciado nas figuras 2 (a) e 2 (b).

Após a análise quantitativa dos comportamentos das temperaturas, é possível realizar uma análise qualitativa com base da distribuição de temperaturas dos termogramas. Esta análise permite identificar regiões com possíveis anomalias térmicas e neste estudo com elementos ocultos inseridos é possível observar na figura 3 alguns dos termogramas obtidos no experimento. São apresentados quatro termogramas sendo a figura 3(a) referente ao início do ciclo de aquecimento, a figura 3(b) o final do ciclo de aquecimento, a figura 3(c) o início do arrefecimento e a figura 3(d) o final do ciclo de arrefecimento. Como resultado das diferenças nas propriedades térmicas, nota-se que é possível identificar a maioria dos defeitos tanto no ciclo de aquecimento quanto arrefecimento. Pode-se observar que os defeitos são mais visíveis ao início do ciclo de aquecimento quando comparados ao final deste ciclo. Isto ocorre pois no começo da etapa de aquecimento a diferença de temperatura entre os elementos ocultos e a argamassa de revestimento é mais evidente, com o avanço do aquecimento é possível observar no termograma final que o comportamento de temperatura da placa e dos defeitos se torna mais uniforme, apesar de apresentar maiores temperaturas. No ciclo de arrefecimento é possível identificar o posicionamento dos defeitos, porém a visibilidade é menor quando comparado ao ciclo de aquecimento, isso ocorre devido a maior intensidade de fluxo no período de aquecimento. 
(a) Início ciclo aquecimento (4 min)

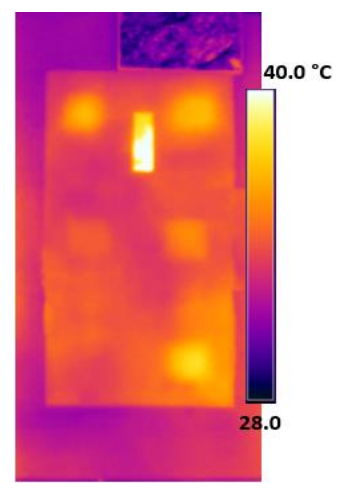

(b) Final ciclo aquecimento (120 min)

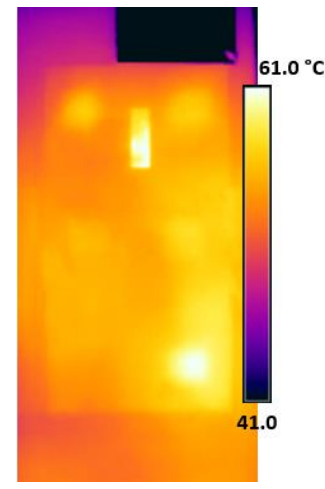

(c) Início ciclo arrefecimento (124 min)

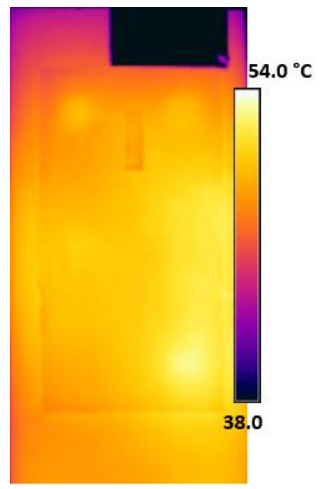

(d) Final ciclo arrefecimento (240 min)

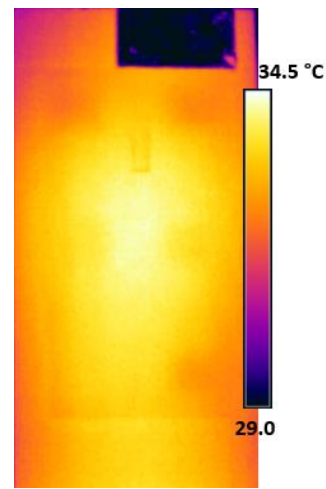

Figura 3 - Termogramas obtidos da placa no ciclo direto nos tempos (a) 4 minutos (b) 120 minutos (c) 124 minutos e (d) 240 minutos (Autores).

Segundo Pavón (2017), considerando o posicionamento dos defeitos sob a camada de revestimento, a condutividade térmica dos materiais é a responsável pela sua visibilidade. Por este motivo os materiais dos defeitos com menores valores de condutividade como o poliestireno, cerâmica e madeira, assim como o vazio que por estar preenchido de ar com condutividade semelhante a do poliestireno, se mostram mais quentes quando comparado ao concreto que apresenta condutividade semelhante a argamassa e por isso se apresenta mais frio em comparação aos demais elementos ocultos. É possível observar no ciclo de aquecimento e arrefecimento que os defeitos mais evidentes são o poliestireno, a madeira e o vazio devido os menores valores de condutividade térmica. Este comportamento de visibilidade em diferentes ciclos do poliestireno como elemento oculto também foi observada nos estudos de Lai et al. (2015), Cheng et al. (2008) e de Weritz et al. (2005).

\section{Conclusões}

Através do experimento realizado pode-se concluir que a aplicação da termografia infravermelha ativa na detecção de elementos ocultos em sistemas de revestimento é satisfatória. A identificação dos defeitos é possível tanto na análise qualitativa quando observado os comportamentos de temperatura dos termogramas quanto na análise quantitativa na determinação do Delta-T.

A condutividade térmica se mostra como parâmetro determinante para identificação dos elementos ocultos nos termogramas na análise qualitativa. Quanto menor o coeficiente de condutividade térmica do material inserido na placa, maior é a dificuldade de transferência de calor através da região do defeito de uma superfície a outra da placa, com isso no ciclo de aquecimento os defeitos se apresentam com temperatura superficial superior a placa e no ciclo de arrefecimento apresentam temperatura superficial inferior a argamassa da placa.

Com isso, considerando os resultados de Delta-T em módulo, os materiais madeira (M), poliestireno expandido $(P)$ e vazio $(V)$ com menor coeficiente de condutividade térmica, quando comparados a argamassa da placa, tendem a apresentar maiores contrastes (Delta- $T$ ) no aquecimento e no arrefecimento. Os materiais com condutividade térmica próxima a da argamassa como o concreto (C), tijolo cerâmico vazado (TV) e tijolo cerâmico maciço (TM), tendem a apresentar menores valores de Delta-T.

\section{Agradecimentos}

O presente trabalho foi realizado com apoio do Conselho Nacional de Desenvolvimento Científico e Tecnológico (CNPq) e do Laboratório de Ensaios de Materiais (LEM-UnB).

\section{Referências Bibliográficas}


ASSOCIAÇÃO BRASILEIRA DE NORMAS TÉCNICAS. ABNT NBR 15220-2 Desempenho térmico de edificações Parte 2: Métodos de cálculo da transmitância térmica, da capacidade térmica, do atraso térmico e do fator solar de elementos e componentes de edificações, 2005.

ASTM E1862-14, Standard Practice for Measuring and Compensating for Reflected Temperature Using Infrared Imaging Radiometers, ASTM International, West Conshohocken, PA, 2014, www.astm.org

ASTM E1933-14, Standard Practice for Measuring and Compensating for Emissivity Using Infrared Imaging Radiometers, ASTM International, West Conshohocken, PA, 2014, www.astm.org.

AIDAR, L.; MILHOMEM, P.; BAUER, E. Análise das possíveis variações nos termogramas provocadas por alterações no ângulo de obtenção de imagens. Congresso Brasileiro de Patologia das Construções CBPAT. Campo Grande, Mato Grosso do Sul . Abril, 2018.

BARREIRA, E.; ALMEIDA, R. M. S. F.; DELGADO, J. M. P. Q. Infrared thermography for assessing moisture related phenomena in building components. Construction and Building Materials, v. 110, p. 251-269, maio 2016.

BAUER, E.; FREITAS, V. P. DE; MUSTELIER, N.; BARREIRA, E.; FREITAS, S. S. DE. Infrared thermography evaluation of the results reproducibility. Structural Survey, v. 31, n. 3, p. 181-193, 2015.

BAUER, E.; PAVON, E. Termografia de infravermelho na identificação e avaliação de manifestações patológicas em edifícios. ISSN 1809-7197. Ano XLIII 79. Jul-Set, 2015.

BAUER, E.; PAVÓN, E.; BARREIRA, E.; CASTRO, E. K. DE. Analysis of building facade defects using infrared thermography: Laboratory studies. Journal of Building Engineering, v. 6, p. 93-104, 2016.

BAUER, E.; PAVÓN, Elier. Criteria for Identification of Ceramic Detachments in Building Facades with Infrared Thermography. Developments in Building Diagnosis Techniques, vol. 5, 2016b, pp. 51-68.

CHENG, C.-C.; CHENG, T.-M.; CHIANG, C.-H. Defect detection of concrete structures using both infrared thermography and elastic waves. Automation in Construction, v. 18, p. 87-92, dez. 2008.

EDIS, E.; FLORES-COLEN, I.; BRITO, J. DE. Passive thermographic detection of moisture problems in façades with adhered ceramic cladding. Construction and Building Materials, v.51, n. 0, p. 187-197, 31 jan. 2014.

EDIS, E.; FLORES-COLEN, I.; BRITO, J. DE. Building Thermography: Detection of Delamination of Adhered Ceramic Claddings Using the Passive Approach. Journal of Nondestructive Evaluation, v. 34, 2015.

ISO/DIS 15686-2: Buildings - Service life planning. Part 2: Service life prediction procedures, International Organization for Standardization, 2012.

LAI, W. W.-L.; LEE, K.-K.; POON, C.-S. Validation of size estimation of debonds in external wall's composite finishes via passive Infrared thermography and a gradient algorithm. Construction and Building Materials, v. 87, p. 113-124, jul. 2015.

MALDAGUE, X. Applications of infrared thermography in nondestructive evaluation. Quebec, Canada. 2001.

MADRUGA, F. J.; IBARRA-CASTANEDO, C.; CONDE, O. M.; LÓPEZ-HIGUERA, J. M.; MALDAGUE, X. Infrared thermography processing based on higher-order statistics. NDT \& E International, v. 43, n. 8, p. 661-666, nov. 2010.

MILHOMEM, M. P. (2019). Critérios para identificação e avaliação do grau de dano de fissuras em fachadas de edifícios com aplicação da termografia de infravermelho. Dissertação de Mestrado em Estruturas e Construção Civil, Publicação 08A/19, Departamento de Engenharia Civil e Ambiental, Universidade de Brasília, Brasília, DF, 115p.

PEDRA, S. A. Potencialidades da termografia infravermelha aplicada ao design do conforto térmico de alvenaria estrutural. Dissertação de mestrado. Universidade de Minas Gerais, 2011.

PAVÓN, E (2017). Critérios e padrões de comportamento para avaliação de descolamentos cerâmicos com termografia de infravermelho. Tese de Doutorado, Publicação E.TD-6A/17, Departamento de Engenharia Civil e Ambiental, Universidade de Brasília, Brasília- DF, 250p.

SILVA, D. D. S. Diagnóstico de patologias em fachadas utilizando termografia. Mestrado. Engenharia Civil. Universidade do Porto, 2012.

WERITZ, F.; ARNDT, R.; RÖLLIG, M.; MAIERHOFER, C.; WIGGENHAUSER, H. Investigation of concrete structures with pulse phase thermography. Materials and Structures, v. 38, n. 283, p. 843-849, 9 mar. 2005.

ZANONI, V.A.G. (2015). Influência dos agentes climáticos de degradação no comportamento higrotérmico de fachadas em Brasília. Tese de Doutorado em Arquitetura e Urbanismo. Programa de Pós-Graduação da Faculdade de Arquitetura e Urbanismo da Universidade de Brasília, Brasília, DF, 293 p. 